\title{
Undifferentiated small cell hepatoblastoma with rhabdoid morphology diagnosed prenatally: case report
}

\begin{abstract}
Liver tumors are rare in children. Hepatoblastoma $(\mathrm{HB})$ is usually diagnosed during the first three years of life and represents approximately $80 \%$ of malignant tumors. Occasionally it can be diagnosed in neonates or during the prenatal period. It is an embryonic tumor that originates from hepatocyte precursors that recapitulate stages of organ development. The variant of undifferentiated small cells or Small Cell Undifferentiated (SCUD), is an epithelial variant of $\mathrm{HB}$ that represents between 3 to $5 \%$ of cases, occurring in a younger age group of 0-1 years old are low or normal serum alpha-fetoprotein unlike the classic variant and exhibits a very aggressive clinical behavior. It is a population of small round and blue cells that co-express stromal and epithelial markers and can grow in a diffuse pattern, in groups, nests or organoid pattern. Histology is a prognostic factor of death risk factor. There is a subgroup of SCUD that exhibits morphological and immunophenotypic characteristic of hepatic malignant rhabdoid tumors such as loss of nuclear expression of INI-1 by immunohistochemistry with or without deletion of the INI gene. It is an extremely aggressive neoplasm with a diffuse growth pattern and should be distinguished from other small and round small blue cell tumors. The objective is to present an infrequent case of embryonic hepatocyte tumor with prenatal presentation.
\end{abstract}

Volume 5 Issue $6-2019$

\author{
Andrea Bosaleh,' María del Valle Centeno,' \\ Victoria Sobrero, ${ }^{2}$ Adriana Rose, ${ }^{2}$ Walter \\ Cacciavillano ${ }^{2}$ \\ 'Department of Pathology, National Hospital of Pediatrics, \\ Argentina \\ ${ }^{2}$ Hemato-Oncology, National Hospital of Pediatrics, Argentina
}

Correspondence: Andrea Bosaleh, Hospital Garrahan. Combate de los Pozos I88I. CABA. CI245 AAM. Buenos Aires, Argentina, Tel 054I I-4I226255/54,

Email andreapaulabosaleh@gmail.com

Received:September 20, 2019 | Published: November 04, 2019

Keywords: hepatoblastoma, small cell, rhabdoid tumor, liver, prenatal

Abbreviations: HB, hepatoblastoma; SCUD, small cell undifferentiated; AFP, alpha-fetoprotein; FISH, fluorescent in situ hybridization; CT, computed axial tomography; INI-1, integrase integrator 1; HPF, high power field; EpSSG NRSTS, European pediatric soft tissue sarcoma study group - non-rhabdomyosarcoma soft tissue sarcomas, MRT, malignant rhabdoid tumors; IHC, immunohistochemistry.

\section{Introduction}

Approximately two thirds of the hepatic masses that occur in children are malignant, with $70 \%$ of them showing hepatocyte origin, both hepatoblastoma (HB) and hepatocarcinoma . HB is diagnosed during the first three years of life and represents approximately $80 \%$ of malignant tumors. Occasionally it can be diagnosed in neonates or during the prenatal period. ${ }^{1}$

It is an embryonic tumor that originates from hepatocyte precursors that recapitulate stages of organ development. It usually demonstrates a combination of epithelial, mesenchymal elements, as well as undifferentiated elements or other heterologous elements to the organ. In 2011, the Children's Oncology Group collaborated with a consensus group of experts that reviewed primary liver tumors and developed a consensus classification including the new reported entities that were published in $2013 .^{2}$ Table 1 shows Classification of HB with its components and histological description.

Table I HB Classification (adapted from Lopez-Terrada)

\begin{tabular}{|c|c|c|}
\hline $\begin{array}{l}\text { HB } \\
\text { (frequency \%) }\end{array}$ & Subtype & Histological description \\
\hline \multirow[t]{5}{*}{ Epitelial (55\%) } & Fetal (F) Well & Uniform cells $(10-20 \mu$ in diameter), round nuclei, cords with minimal mitotic \\
\hline & differentiated: & activity (<2mitosis/IOHPF), conspicuous nucleoli, les glycogen. \\
\hline & Fetal $(F)$ & Moderate anisonucleosis, high N/C, nucleoli, anaplastic: marked nuclear \\
\hline & Pleomorphic or & enlargement and pleomorphism, hyperchromatic, abnormal mitosis nucleoli \\
\hline & poorly & and high $\mathrm{N} / \mathrm{C}$ \\
\hline $30 \%$ & differentiated & \\
\hline \multirow[t]{2}{*}{$20 \%$} & Embrionario(E) & $10-15 \mu$ in diameter, high N/C, angulated nuclei, primitive tubules, \\
\hline & & extramedullary hemopoiesis. \\
\hline \multirow[t]{2}{*}{$3-5 \%$} & Macro trabecular & Epithelial HB F or E growing in trabeculae $>5$ cells thick (between sinusoids) \\
\hline & (MT) & \\
\hline
\end{tabular}


Table Continued...

\begin{tabular}{lll}
\hline $\begin{array}{l}\text { HB } \\
\text { (frequency \%) }\end{array}$ & Subtype & Histological description \\
\hline $3-5 \%$ & Small Cell & $5-10 \mu$ in diameter, no architectural pattern, pale amphophilic cytoplasm, round \\
& undifferentiated & to oval nuclei with fine chromatin and inconspicuous nucleoli, +/- mitosis, $+/-$ \\
& (SCUD) & INII \\
& Cholangioblastic & Bile ducts, usually at periphery of epithelial islands, can predominate \\
& Stromal & Células fusiformes (blastema), osteoide, musculo esquelético, cartílago \\
Mixed & derivatives & \\
$45 \%$ & Teratoid & Mixed, plus primitive endoderm, neural derivatives, melanin, squamous and \\
& & glandular elements. \\
\hline
\end{tabular}

In our country, according to data from Argentine Oncopediatric Hospitalary Registry (ROHA), HB has an incidence of 1.03 cases per 1,000,000 inhabitants under 15 years, with a male: female ratio of 1.3: 1 and an annual average of 18 annual cases in the country. (Data 2000-2016). ${ }^{3}$ Some genetic syndromes such as familial adenomatous polyposis, Beckwith-Wiedemann syndrome and less frequently trisomy 18, the syndromes of Simpson-Golabi- Behmel, Prader-Willi, Sotos, have been identified as predisposing factors for HB. In addition, there is evidence that children with very low birth weight under 1,500 gr. have a twenty-fold greater risk of suffering from HB than the general population. Complete surgery, whether due to surgical resection of the tumor, or liver transplantation, is essential in the treatment of $\mathrm{HB}$, being the most important prognostic factor in children with this pathology. Currently, the classic epithelial and mixed variants have a survival rate of approximately $75-80 \%$.

The variant of undifferentiated small cells or Small Cell Undifferentiated (SCUD), is an epithelial variant of HB that occurs between 3 to $5 \%$ of cases, may occur associated with other classic variants or in pure form when It represents more than $75 \%$ of the tumor mass. It is a population of small round and blue cells that co-express stromal and epithelial markers such as vimentin and cytokeratin, being negative for Alpha-fetus protein (AFP) and glipican-3, can grow in a diffuse pattern, in groups, nests or organoid pattern. Clinically presented in a younger age group of $0-1$ years old are low or normal serum AFP unlike the classic variant and exhibits a very aggressive clinical behavior. ${ }^{1}$ Histology is a prognostic factor of death risk factor. There is a subgroup of SCUD that exhibits morphological and immunophenotypic characteristic of hepatic malignant rhabdoid tumors (MRT) such as loss of nuclear expression of INI-1 by immunohistochemistry with or without deletion of the INI gene and transition between these two entities have been described.

The objective is to present an infrequent case of embryonic hepatocyte tumor, of prenatal presentation with SCUD morphology and rhabdoid pattern with loss of nuclear expression of INI -1 without deletion by fluorescent in situ hybridization (FISH).

\section{Case report}

\section{Clinical data}

We present a male patient, new born term, with high weight according to gestational age. No inherited history of importance. Maternal pregnancy controlled, in the 3rd trimester of pregnancy, liver mass ultrasound was detected that compromised the right liver lobe, segments VI and VII. Predominantly cystic image of net and lobed edges of $20 \times 21 \mathrm{~mm}$ with echoes inside and papillary projections on its wall. At 30 days of life a solid-cystic mass of $5.8 \times 4.5 \times 4.4 \mathrm{~cm}$ $\left(\right.$ Vol. $\left.59 \mathrm{~cm}^{3}\right)$ was observed on abdominal ultrasound, without vascular involvement. Computed axial tomography (CT) of the abdomen was performed in which well-defined cystic formation was observed, polylobed with fine septa inside measuring $5.7 \times 5.5 \times 5 \mathrm{~cm}$ (Vol. $81 \mathrm{~cm}^{3}$ ), compromising the right liver lobe (segment V, VI, VII and VIII). (Figure 1) Chest CT without evidence of disease. Dosage of AFP and human chorionic gonadotrophin with normal values. Due to the age and the findings of complementary studies, having ruled out vascular etiology, at 75 days of life, tumor resection was performed. Surgery had been delayed 1 month due to intercurrent infection.

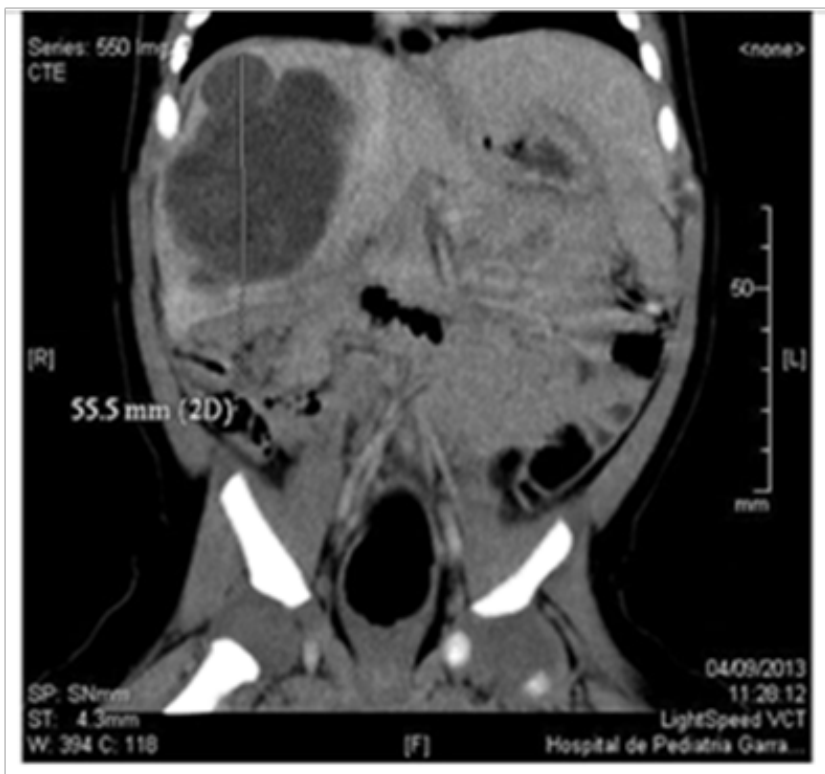

Figure I Abdominal CT with tumor injury that compromises the right liver lobe at the level of segment V,VI, VII and VIII.

\section{Histopathology}

Right hepatectomy (segment V, VI, VII and VIII) of $11 \times 9 \mathrm{~cm}$ with a weight of $380 \mathrm{grs}$. The cut was solid-cystic multilobed tumor formation of $9.5 \times 6.8 \times 6 \mathrm{~cm}$. It was $0.1 \mathrm{~cm}$ from the surgical margin of the resection (Figure 2). It was set to formal aldehyde at $10 \%$. It was colored with $\mathrm{H}-\mathrm{E}$ and immunohistochemical, and FISH techniques 
with LSI22 (BCR) probe were performed. Histologically solid neoplastic proliferation, consisting of atypical cells, on solid beaches, in nests and laces. (Figure 3) Areas with vesicular, irregular nuclei with nucleoli and others with eosophilic cytoplasm and evident rhabdoidtype nucleus. (Figure 4) Mitosis up to 8/10 high power field (HPF) and presence of multiple vasculo-lymphatic embolisms. (Figure 5) No elements of classic HB were observed. Lymph node with metastasis. (0/1) Presence of extramedullary hemopoiesis.

The following markers were positive: Vimentin (Figure 6), cytokeratin: CK (AE1-AE3) (Figure 7) and membrane epithelial antigen. Integrase Integrator 1 (INI-1) demonstrated loss of nuclear expression with respected nuclear marking in endothelial cells and lymphocytes (Figure 8), Beta-catenin, Hepar-1, glypican 3, desmin and Myf- 4 were negative. Proliferation fraction Ki-67 was $50 \%$ (Figure 9). FISH was performed with the LSI22 probe (BCR) specific to the chromosomal region 22q11.2 8gen (INI-1) on paraffine block with negative results.

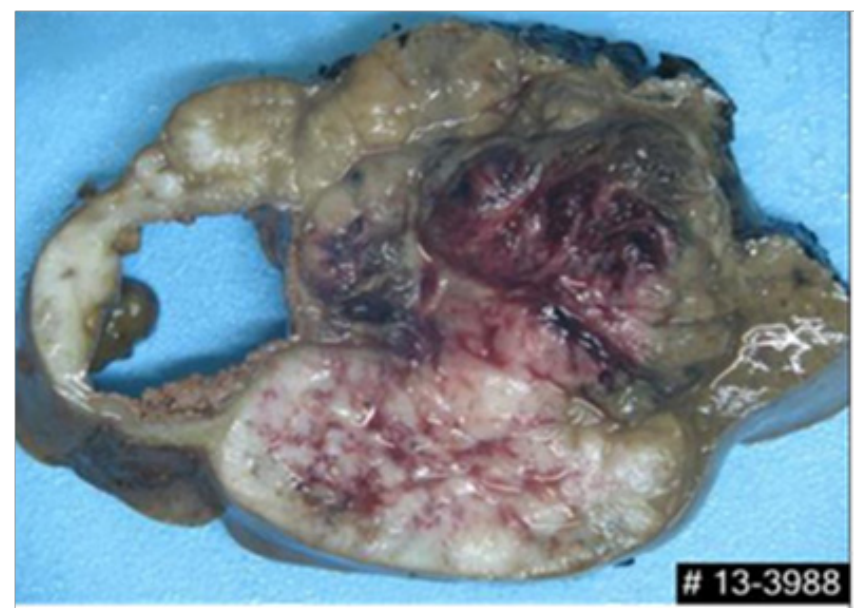

Figure 2 Hepatectomy with cystic solid tumor formation, whitish with hemorrhagic and fleshy areas.

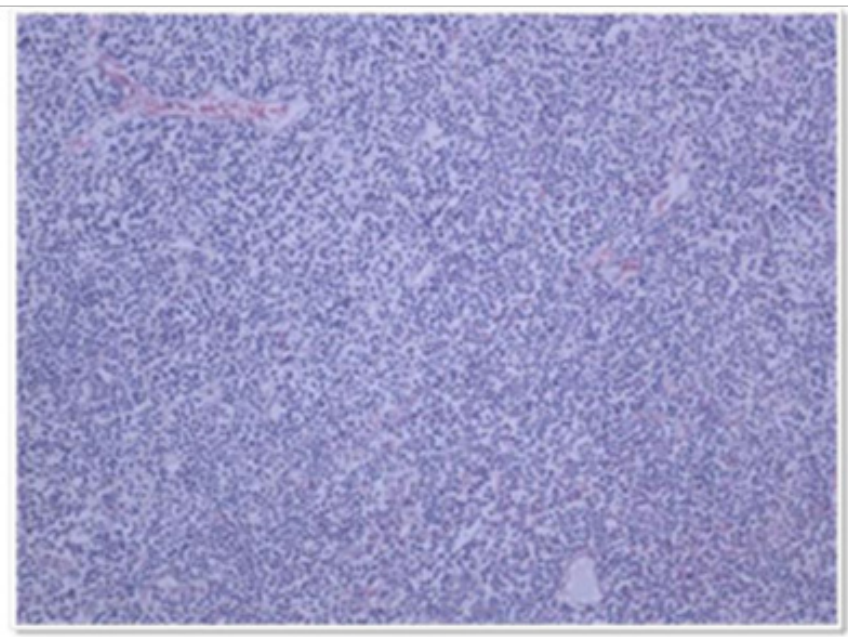

Figure $3 \mathrm{H}-\mathrm{E}$ : I00X: Solid neoplastic proliferation, consisting of atypical cells, on solid pattern.

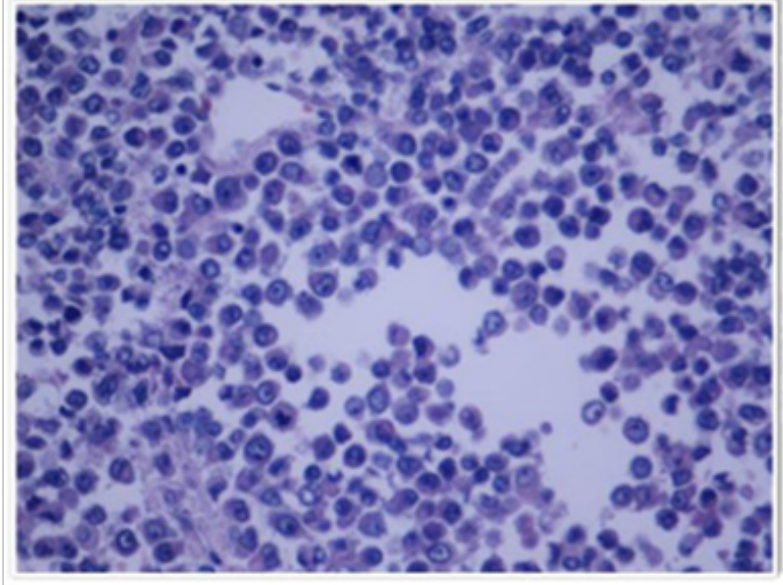

Figure $4 \mathrm{H}-\mathrm{E}$ 400X. Areas with vesicular, irregular nuclei.

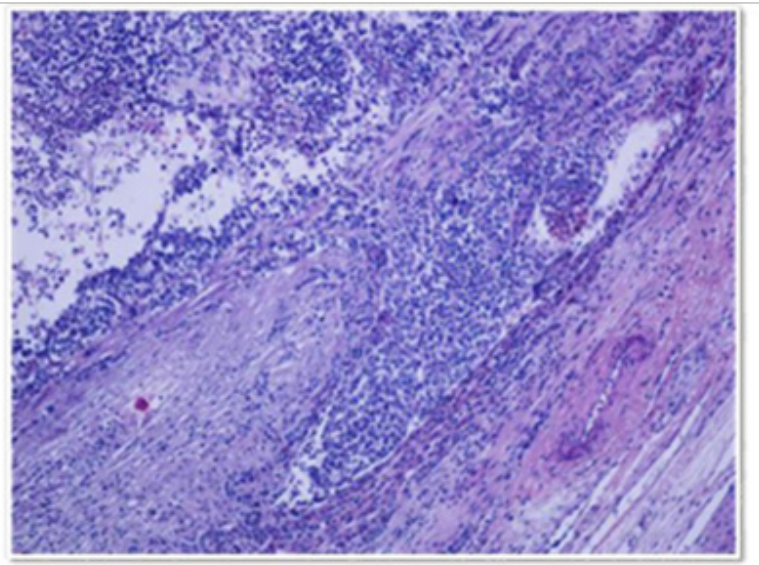

Figure 5 H-E 400X: Vascular embolias with nucleolus and others with eosinophilic cytoplasm and evident rhabdoid-type.

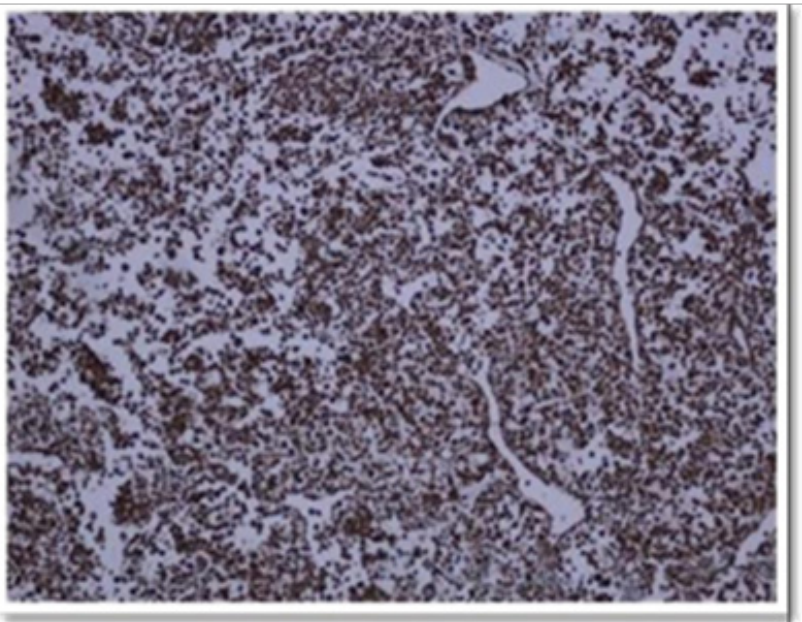

Figure 6 IHC.Vimentin I00X. 


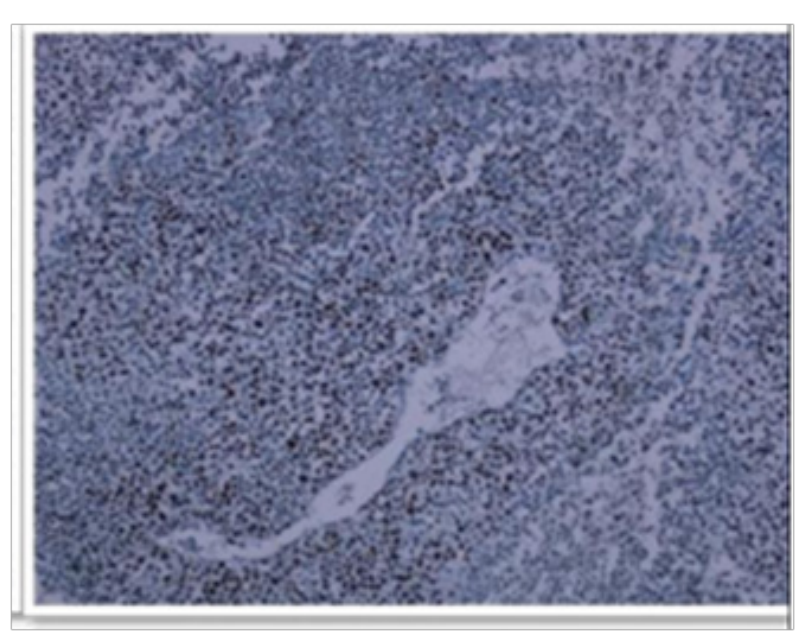

Figure 7 IHC CK (AEI-AE3) I00X.

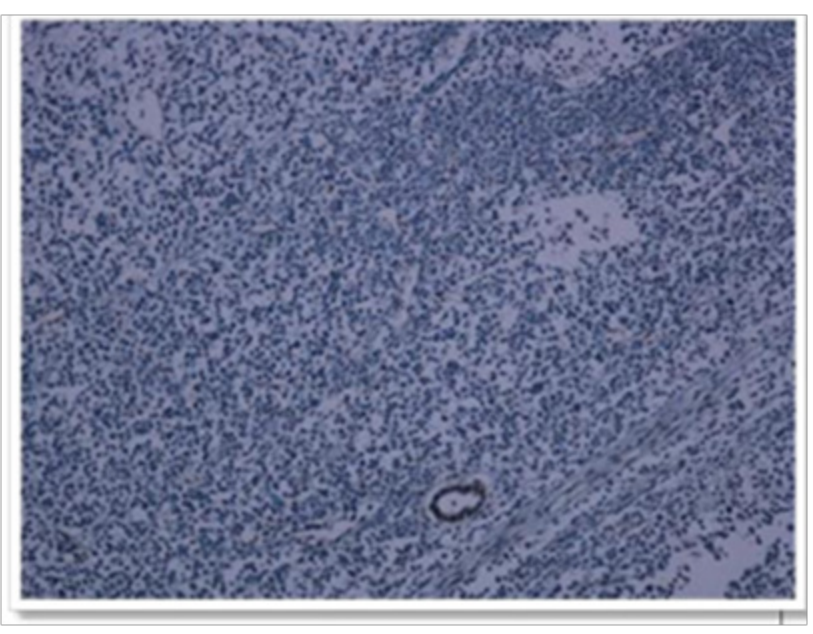

Figure $8 \mathrm{IHC}$.INI-I 100X. loss of nuclear expression

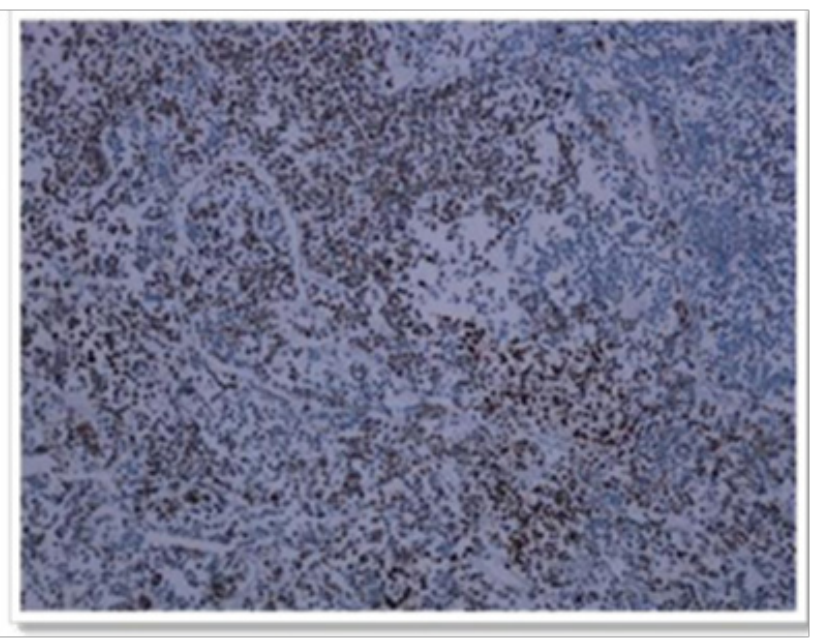

Figure 9 IHC. Ki-67: 100X.

\section{Post-surgery staging}

A total body scan with Technetium- ${ }^{99}$ was performed that resulted normal, and chest CT showed multiple bilateral lung metastasis, with normal brain CT. With preliminary diagnosis of Rhabdoid Liver
Tumor, the patient received EpSSG NRSTS 2005 protocol. After the first cycle the patient developed metastatic lung disease progression, dying within two months of diagnosis.

\section{Discussion}

We present a rare prenatal tumor consisting of small undifferentiated cells with loss of protein expression of the INI-1 protein with $22 \mathrm{q} 11.2$ negative chromosome region removal by FISH. Within tumors with immunostaining-negative INI-1 are HB-SCUD with rhabdoid morphology and MRT.

HB-SCUD is a subgroup of HB that represents less than $5 \%$ of them and is characterized by a population of undifferentiated cells that mimic other small, round and blue cell tumors. It is an extremely aggressive neoplasm with a diffuse growth pattern. These cells had previously been described by Misugi et al. ${ }^{4}$ Haas $^{5}$ proposed changing the name of anaplasia to that of undifferentiated small cells of today. They were subsequently described by González Cruss, ${ }^{6}$ Hansen in $1992^{7}$ and Stocker. ${ }^{8}$

It is a highly aggressive fast-growing tumor with an estimated 2-year survival that does not exceed $0 \% .{ }^{5}$ Few reports have analyzed clinical behavior primarily related to their rarity. Trobaugh-Lotario et al., ${ }^{9}$ described 11 patients, 10 of whom died from progression and 1 from complications of treatment. Six of the six evaluated were INI-1 negative by immunostaining and one case evaluated showed cytogenetic and molecular abnormalities similar to malignant rhabdoid tumors (MRTs).

Histologically they are characterized by a population of small ovoid, starry or fusiform cells with diffuse or nodular growth pattern. The nuclei exhibit dense chromatin. The tumor shows an infiltrative edge and shows prominent intravascular growth. Necrosis can be extensive. Within the SCUD Zimmerman ${ }^{1}$ describes three types of SCUDs. Immunohistochemistry: they are reagents for vimentin, pan-cytoqueratin with high fraction of proliferation with Ki67. They usually do not express AFP. ${ }^{1}$ There is a subgroup of HB-SCUD shave with rhabdoid characteristics that would appear to be related to liver MRT. MRTs are highly aggressive tumors that usually occur in the CNS and kidney in infants and children. Less frequently they can arrive at other sites including soft tissues, abdomen, pelvis, retroperitoneum, heart and gastrointestinal tract. Also described in the liver. ${ }^{10-14}$ Histologically there are characterized by large polygonal cell plates with abundant cytoplasm and vesicular nucleus with prominent central nucleoli. Rhabdoid cells show eosiphilic or amphophilic cytoplasm containing perinuclear cytoplasmic inclusions consisting of intermediate filaments positive for epithelial markers and mesenchymal as vimentin. An important but most subgroup are characterized by deletions of region 11.2..of the long arm of chromosome 22 (22q11.2) and show frameshift or non-sense mutations of INI1, detectable by the loss of protein expression of INI1.

\section{Conclusion}

We present a newborn with a prenatal diagnosis of liver tumor with subsequent diagnosis of SCUD-HB with focal rhabdoid morphology with nodal metastases at diagnosis and rapid massive pulmonary metastatic evolution. SCUD are uncommon accounting for $5 \% \mathrm{HB}$, with isolated cases of prenatal presentation. The subgroup of HBSCUD with rhabdoid characteristics INI-I1 negative should be called tumors "with rhabdoid characteristics". Is important to identify those patients who require aggressive therapy. 
The presence of SCUD in a HB is associated with adverse prognosis and would appear to be biologically different from the nonSCUD. From a histological point of view, the recommendations are the documentation of the percentage of the SCUD component and the evaluation of the recommended IHC panel and molecular test. It is important to look for areas of classical HB to certify the diagnosis and rule out MTR. More extensive comparative clinic-pathologic and molecular analysis will be help to clarify differences between rhabdoid tumors and the SCUD.

\section{Acknowledgments}

Thanks to Dr. Jessica Lopez Marti coordinator for the area of molecular and Dora Diaz histotechnics of the molecular area. (FISH)

\section{Conflicts of interest}

I do not have conflicts of interest for the publication of this article and no one has funded it.

\section{Funding details}

None.

\section{References}

1. Zimmermann A, Lopez-Terrada D. Pediatric Liver Tumors. Pediatric Oncology. 2011. p. 83-112.

2. Lopez Terrada D, Alaggio R, de Davila MT, et al. Methods in Pathology. Toward an International pediatric liver tumor consensus classification: proceedings of the Los Angeles COG liver tumors symposium. Modern Pathology. 2013. p. 472-491.

3. Argentine Hospital Oncopediatric Registry: Incidence Trend 20002016. Secular survival trend: 2000-04, 2005-09 and 2010-14. 6th edn. Autonomous City of Buenos Aires: National Cancer Institute, 2018.

4. Misugi K, Okijima H, NisugiN, et al. Classification of primary malignant tumors of liver in infancy and childhood. Cancer. 1967;20:1760-1771.
5. Haas JE, Muczynsky KA, Krailo M, et al. Histopathology and prognosis in childhood Hepatoblastoma and Hepatocarcinomas. Cancer. 1989;64:1082-1095.

6. Gonzalez-Crussi F. Undifferentiated small cell ("anaplastic") hepatoblastoma. Pediatr Pathol. 1991;11:155-161.

7. Hansen K, Bagtas J, Mark HF, et al. Undifferentiated small cell Hepatoblastoma with a unique chromosomal translocation: A case report. Pediatr Path. 1992;12:457-462.

8. Stocker JT. Hepatoblastoma. Semin Diagn Pathol. 1994;11:136-143.

9. Trobaugh-LotarioA, Tomlinson G, Finegold M, et al. Small Cell Undifferentiated Variant of Hepatoblastoma: Adverse Clinical and Molecular Features Similar to Rhabdoid Tumors. Pediatr Blood Cancer. 2009;52(3):328-334.

10. Vokuhi C, Oyen F, Haberle B, et al. Small Cell Undifferentiated (SCUD) Hepatoblastomas: All Malignant Rhabdoid Tumors. Genes Chromosomes \& Cancer. 2016:1-7.

11. Nguyen H, Stelling A, Kuramoto A, et al. Malignant rhabdoid tumors of the liver: Findings at US, CT, and MRI with histopathologic correlation. RCR. 20114;1:1-3.

12. Parham DM, Weeks DA, Beckwith JB. The clinicopathologic spectrum of putative extrarenal rhabdoid tumors. An analysis of 42 cases studied with immunohistochemistry or electron microscopy. Am J Surg Patol. 1994;18:1010-1029.

13. Scheimberg I, Cullinane C, Kelsey A, et al. Primary hepatic malignant tumor with rhabdoid features. A histological, immunocytochemical, and electron microscopic study of four cases and a review of the literature. Am J Surg Pathol. 1996;20:1394-1400.

14. Garces-Iñigo EF, Leung R, Sebire NJ, et al. Extrarenal rhabdoid tumours outside the central nervous system in infancy. Pediatr Radiol. 2009;39:817-822. 